\title{
Assessing Cultural Sensitivity Questions in Ranking Decisions for a Family Medicine Residency Program
}

\author{
Lori Schramm ${ }^{1}$, Adam Clay $^{1}$, Brian Geller ${ }^{1}$ \\ ${ }^{1}$ Department of Academic Family Medicine, University of Saskatchewan, Canada \\ *Corresponding author. Email: lls651@usask.ca
}

\begin{abstract}
Background and aims/objectives: The ability of family physicians to establish an inclusive and culturally-safe practice environment is a key competency of the profession. Our study aimed to explore the utility of questions assessing cultural sensitivity to improve ranking decisions of family medicine residency candidates. Methods: A series of cross-sectional online surveys were sent to interviewers (current residents and faculty), site directors and administrators, following completion of the first period of national resident interview dates. The surveys contained both closed and open-ended questions about the utility of cultural sensitivity questions during the interview process. Frequency distributions were calculated in Microsoft Excel for the 5-point Likert items. Open-ended data was themed by an independent researcher. This project was exempted by the Behavioural Research Ethics Board of the University of Saskatchewan. Results: The majority of respondents felt the questions helped them identify candidates that would fit the program. Local modifications were done to adapt to local context or improve clarity. For example, questions were generalized to vulnerable populations or narrowed specifically to experiences with Indigenous populations. Some participants indicated that cultural knowledge, as opposed to empathy, can be taught and thus the latter is what the assessment of candidates' abilities should focus on. It was also suggested that these questions detract from opportunities to assess "particularly relevant clinical experiences or personal experiences outside of medicine." Conclusions and Recommendation: Interviewers generally felt cultural sensitivity questions improved ranking decisions. Additionally, allowing flexibility to adapt to local contexts was important. Future initiatives can focus interviewer training on cultural sensitivity/safety approaches.
\end{abstract}

Keywords: Candidate selection, cultural sensitivity.

\section{INTRODUCTION}

Research has shown many health inequities between Indigenous and non-Indigenous populations in Canada, Australia and other nations with colonial histories [1]. Building relationships of cultural understanding and trust between healthcare providers and Indigenous peoples is one of the best ways to support healing of individuals and communities [2]. The Truth and Reconciliation Commission of Canada: Calls to Action stress the importance of cultural safe healthcare in closing the gaps in health outcomes [3]. Other population groups such as, but not limited to, Black or African Canadians, individuals identifying with the LGBTQ2+ community, certain religious groups, or people living with addictions also disproportionately experience poorer healthcare outcomes and would benefit from culturally safe healthcare provision that considers the social and historical contexts of specific population groups [4].
Cultural competency and safety considerations have been introduced into healthcare practice standards in Canada [5]. For example, CanMED-Family Medicine outlines the core competency framework designed for all Canadian family physicians regardless of practice type, location, or populations served. Cultural sensitivity training is included as part of undergraduate medical education in Canada. As such, medical school graduates seeking residency positions should have prior exposure to culturally safe care. Establishing an inclusive and culturally-safe practice environment is a key competency identified [6]. However, racism towards Indigenous populations continues to be a major concern in the Canadian healthcare system. These biases towards Indigenous patients impacts quality of care [7] and the willingness of patients to seek medical care [8]. Canadians who identify as African, Caribbean, or Black, LGBTQ2+, or those living with mental illness, substance use disorders, HIV or obesity also experience stigma and discrimination in healthcare which contributes to health inequities [4]. 
Each winter, invited applicants to Family Medicine residency programs across Canada undergo interviews as part of the selection process, facilitated by the Canadian Resident Matching Service (CaRMS) organization. Applicants are matched to residency programs based on a combination of a programs' rankings of interviewed candidates and applicants' own rankings of site preference.

As part of the interview process, faculty members and current residents ask candidates a range of questions in order to assess their abilities along numerous domains. These include candidates' compatibility with the site/program, their learning goals and objectives, medical knowledge, personal strengths and weaknesses, critical thinking and problem solving, emotional intelligence and maturity, and interpersonal skills.

The University of Saskatchewan accepts approximately 50 new family medicine residents each year. The residents are geographically located across the province at seven training sites. In 2019, four questions assessing cultural sensitivity were added to the behavioural interview protocol for family medicine residency positions at the University of Saskatchewan.

The cultural sensitivity questions were designed by a certified family physician following review of the grey literature and discussions with various interested and informed parties. The final questions were reviewed and approved by the Department's Residency Program Committee. Sites did not have to ask all the cultural sensitivity questions and could choose their preferred question(s). Candidate responses were assessed using a scoring scale that was provided to each interviewer. Each candidate was evaluated based on the STAR method (ability to describe the situation, tasks required, actions used and result of their actions) for behavioural style interview questions. Candidates were scored out of 10 for the cultural sensitivity questions, with the overall interview score out of 60 .

The objective of this study was to assess how interviewers perceived the quality and utility of the cultural sensitivity questions.

\section{METHODS}

Design: Several brief cross-sectional online surveys, hosted in SurveyMonkey, were distributed by the Administrative Assistant to the Postgraduate Director of Family Medicine. Surveys were distributed following the completion of the first round of candidate interviews.

The surveys asked items related to the cultural sensitivity questions asked during candidate interviews. These surveys were distributed in both 2019 and 2020 to all interviewers at the DAFM training sites. The surveys consisted of a close-ended 5-point Likert items and openended questions.
Analysis: Frequency distributions were calculated in Microsoft Excel for the 5-point Likert items. Open-ended data was themed by two independent researchers.

This project was reviewed and exempted by the Behavioral Research Ethics Board of the University of Saskatchewan.

\section{RESULTS \\ 3.1 Quantitative data}

Respondent demographics: The 2019 cultural sensitivity survey was completed by 24 faculty members and 22 residents. One individual did not indicate whether they were a faculty member or resident. Of these 47 respondents, approximately half (53\%) were from rural and regional sites, with an additional 22 (47\%) from urban sites.

The 2020 cultural sensitivity survey was completed by 40 participants. Due to changes to the interview format in 2019, not all interviewers asked cultural sensitivity questions during interviews. As a result, only 23 of these participants asked cultural sensitivity questions and were included in further analysis. Of these 23 participants, $11(48 \%)$ were faculty members and 12 $(52 \%)$ were residents; Twelve respondents $(52 \%)$ were from rural and regional sites, with an additional 11 (48\%) respondents coming from urban sites.

\begin{tabular}{|c|c|}
\hline Question & Frequency $(\%)$ \\
\hline $\begin{array}{l}\text { Tell us about systemic or societal barriers you encountered in working with an } \\
\text { Indigenous patient and how you provided patient-centered and culturally gafe care } \\
\text { in this instance? If you have not worked with Indigenous patients, please describe } \\
\text { an encounter with a patient from a cultural background different than your orn } \\
\text { dominant culture or a member of an underserved population. }\end{array}$ & $13(57 \%)$ \\
\hline $\begin{array}{l}\text { Describe an interaction you witnessed where comments were made that were } \\
\text { prejudicial or culturally unsafe. How did you respond? }\end{array}$ & $11(48 \%)$ \\
\hline $\begin{array}{l}\text { Tell us about a time when you adapted your approach to work more effectively } \\
\text { with a person from a different background. }\end{array}$ & $8(35 \%)$ \\
\hline $\begin{array}{l}\text { Talk about a time that you successfully adapted to a culurally different } \\
\text { enviroument. }\end{array}$ & $9(39 \%)$ \\
\hline
\end{tabular}

Cultural sensitivity questions used: In 2020, but not 2019, participants were asked which questions were used at their site. All four cultural sensitivity questions were utilized in urban sites, but smaller sites selected one or two cultural sensitivity questions to ask candidates. The frequencies of question use are provided in Table 1, although some sites did not use these questions verbatim. 
Table 2: Reviewers impression of cultural sensitivity questions.

\begin{tabular}{|l|l|l|l|l|l|}
\hline Survey item & $\begin{array}{l}\text { Strongly } \\
\text { Disagree }\end{array}$ & Disagree & Neutral & Agree & Strongly \\
Agree \\
\hline $\begin{array}{l}\text { I understood what was } \\
\text { meant by the }\end{array}$ & $0(0 \%)$ & $4(6 \%)$ & $8(12 \%)$ & $\begin{array}{c}37 \\
(71 \%)\end{array}$ & $7(11 \%)$ \\
questions & & & & & \\
\hline $\begin{array}{l}\text { The answers provided } \\
\text { by the interviewees } \\
\text { suggested that they } \\
\text { understood what was } \\
\text { being asked of them }\end{array}$ & $3(5 \%)$ & $19(28 \%)$ & $15(22 \%)$ & $\begin{array}{c}26 \\
(39 \%)\end{array}$ & $4(6 \%)$ \\
\hline $\begin{array}{l}\text { These questions } \\
\text { helped me } \\
\text { differentiate } \\
\text { candidates to identify } \\
\text { the best fit for my } \\
\text { program. }\end{array}$ & $2(3 \%)$ & $10(15 \%)$ & $20(30 \%)$ & $\begin{array}{c}30 \\
(45 \%)\end{array}$ & $64(6 \%)$ \\
\hline
\end{tabular}

Understanding and effectiveness of questions: The majority of respondents felt the cultural sensitivity questions were understood by both the applicant and interviewer (Table 2). Similarly, the majority of respondents felt the questions helped them identify candidates that would fit the program. Of note, some respondents strongly disagreed with the statement that the questions helped identify a good fit for their program.

Scoring schema/rubric: In 2020, 13 out of 20 (65\%) respondents found the scoring schema/rubric for the cultural sensitivity questions helpful.

\subsection{Qualitative Data}

\subsubsection{Feedback on Question Design}

The sites reported minor changes to supplied questions, although one faculty member from an urban site reported that they were supplied with a different, "better" set of unspecified questions. When questions were modified, it was usually to adapt to local context or to improve clarity and delivery. For example, questions were generalized to include other vulnerable populations or narrowed by only asking questions that specified Indigenous populations.

\subsubsection{Cultural Safety and Awareness as a Competency}

Participants who provided written responses pertaining to the issue of cultural sensitivity as a competency within Family Medicine were largely unsupportive of including questions specifically addressing scenarios involving Indigenous patients and did not view this as its own area of competency. Some participants indicated that cultural knowledge, as opposed to empathy, can be taught and thus, the latter should be the focus for assessing candidates' abilities. It was also suggested that these questions detract from opportunities to assess "particularly relevant clinical experiences or personal experiences outside of medicine."
Concerns were raised about why Indigenous patients in particular were the focus of these questions, with several indicating that all patients deserve equality and respect; however, one respondent specifically noted that these questions help to address an important area. Two respondents noted that including these questions reflect the University of Saskatchewan's values and demonstrates that Indigenous health is important to this program.

\subsubsection{Value of Questions}

Qualitative comments show the value of the four questions themselves were given a mixed reception. Some participants mentioned that the questions allowed interviewers to rule out some candidates or to recognize bias. Having a patient-family advisor on the interview panel was given as a way to improve the scoring of the question. Of particular concern was a comment provided by a rural resident regarding the attitude of faculty at this person's training site: It was actually the faculty that disappointed me with these questions. They made rude comments about "why do we have to do this" and "pushing the agenda again" type of thing. I don't remember exactly what they said. But I was saddened by the faculty comments. I stood up for these questions and they came around though. Especially after they got such great answers.

The ability of the questions to identify better practitioners was questioned. One urban-based resident stated: No evidence was provided to show that people who score well on this question provide better care.

\section{DISCUSSION}

The value of questions on cultural sensitivity and the need for competency in this area was questioned by several respondents. This is despite CanMEDS-Family Medicine identifying the ability of family physicians to establish an inclusive and culturally-safe practice environment as a key competency [5]. The inclusion of mandatory cultural sensitivity questions may require interviewers to confront their own biases and signal to applicants that the program is working toward inclusion and addressing bias. It also provides an opportunity to explore the applicant's approach to recognizing their own cultural biases and its impact in cross cultural interactions. A MMI format may help further reduce bias as others have reported that MMIs are largely free from cultural bias [9]. Data from a recent survey of incoming family medicine residents in Saskatchewan (unpublished data) show that residents entering the program have lower confidence working with Indigenous populations than with people of other cultures or faiths. Thus, targeted questioning related to experience with Indigenous populations may be relevant. 
There was a perception that evidence was lacking connecting the ability of candidates to answer the cultural sensitivity questions to better patient outcomes. While this is true, demonstrating this effect is challenging and unlikely to occur. However, various studies have demonstrated that increased cultural competence improves patient outcomes and cultural sensitivity and competency skills are required to meet the CanMEDs key competency of providing culturally safe care [10].

While the surveys focused on cultural sensitivity questions the interview process, this was not a controlled experiment and several limitations exist. Responder bias and several confounding factors are present. For example, there are continuous efforts to improve the resident matching process at each site beyond the interview question design and process, which invariably impacts interviewer perception. We also did not measure the student/applicant satisfaction with the interview questions.

Further research should include education initiatives regarding scoring of behavioural style interview questions, and specifically questions assessing for cultural sensitivity. The idea of a patient-family advisor on the interview panel could be explored, particularly as we move towards centralized interviews. This is especially important as culturally safe care is determined to be safe by those receiving the care. Future research and discussion can explore options to incorporate local context into both the cultural sensitivity questions and the interview process. The candidate's experience with the interview process can be a subject of future research.

\section{Conclusion}

Interviewers generally felt cultural sensitivity questions improved ranking decisions. Additionally, allowing flexibility to adapt to local contexts was important. Future initiatives can focus interviewer training on cultural sensitivity/safety approaches.

\section{AUTHORS' CONTRIBUTIONS}

Lori Schramm and Brian Geller contributed to the study conception and design. Data analysis was performed by Adam Clay and Lori Schramm. The first draft of the manuscript was written by Adam Clay and all authors commented on previous versions of the manuscript.

\section{ACKNOWLEDGMENTS}

The authors would like to acknowledge the following contributors:

- Former alumni of the Indigenous Family Medicine residency program at $\mathrm{UBC}$
- Members of the University of Saskatchewan Department of Family Medicine RPC committee

- Members of the Wellness Wheel team

- Dr. Peter Butt, member of the University of Saskatchewan Indigenous Health Committee

- Current faculty from the University of Saskatchewan Family Medicine Residency program involved in equity approaches and vulnerable and underserved curriculum

\section{REFERENCES}

[1] Mitrou, F., Cooke, M., Lawrence, D., Povah, D., Mobilia, E., Guimond, E., \& Zubrick, S. R. (2014). Gaps in Indigenous disadvantage not closing: a census cohort study of social determinants of health in Australia, Canada, and New Zealand from 19812006. BMC Public Health, 14(1), 1-9.

[2] Papps, E., \& Ramsden, I. (1996). Cultural safety in nursing: The New Zealand experience. International Journal for Quality in Health Care, 8(5), 491-497.

[3] Truth and Reconciliation Commission of Canada, (2012) Truth and Reconciliation Commission of Canada: Calls to Action, Exhibits, accessed February 9, 2021, https://exhibits.library.utoronto.ca/items/show/242 $\underline{0}$.

[4] Public Health Agency of Canada (2019) Chief Public Health Officer's Report on the State of Public Health in Canada 2019: Addressing Stigma Towards a More Inclusive Health System. Accessed February 26, 2021, https://www.canada.ca/en/publichealth/corporate/publications/chief-public-healthofficer-reports-state-public-healthcanada/addressing-stigma-toward-more-inclusivehealth-system.html\#a2

[5] Berg, K., McLane, P., Eshkakogan, N., Mantha, J., Lee, T., Crowshoe, C., \& Phillips, A. (2019). Perspectives on Indigenous cultural competency and safety in Canadian hospital emergency departments: A scoping review. International Emergency Nursing, 43, 133-140.

[6] Shaw E, Oandasan I, Fowler N, eds. (2017) CanMEDS-FM 2017: A competency framework for family physicians across the continuum. Mississauga, ON: The College of Family Physicians of Canada.

[7] Nelson, A. (2002). Unequal treatment: confronting racial and ethnic disparities in health care. Journal of the National Medical Association, 94(8), 666.

[8] Browne, A. J., Smye, V. L., Rodney, P., Tang, S. Y., Mussell, B., \& O'Neil, J. (2011). Access to primary 
care from the perspective of Aboriginal patients at an urban emergency department. Qualitative Health Research, 21(3), 333-348.

[9] Hofmeister M., Lockyer J., Crutcher R. (2008) The acceptability of the multiple mini interview for resident selection. Family Medicine, 40(10):73440.

[10] Ward C., Branch C., Fridkin A. (2016) What is Indigenous Cultural Safety-and Why Should I Care About It?. Visions Journal, 11 (4), 29-32

[11] Mesa Community College. Sample Cultural Competency Interview Questions. Accessed February 28, 2021, https://www.mesacc.edu/sites/default/files/pages/se ction/employees/human-

resources/Sample\%20Cultural\%20Competency\%2 OInterview\%20Qustions.pdf 